\title{
PRIMER ESTUDIO DE LA VARIABILIDAD GENÉTICA DE LA ARAHUANA Osteoglossum bicirrhosum (Cuvier, 1829) EN LA REGIÓN LORETO (AMAZONÍA PERUANA)
}

\author{
Werner Chota-Macuyama ${ }^{2,4}$, Carmen García-Dávila ${ }^{1,4}$, Adela Ruiz ${ }^{2,4}$, Fabrice Duponchelle ${ }^{3,4}$, Diana Castro- \\ Ruiz $^{1,4}$, Fred Chu-Koo ${ }^{2,4}$, Jean-François Renno, \\ 1 Laboratorio de Biología y Genética Molecular (LBGM), Instituto de Investigaciones de la Amazonía Peruana (IIAP), \\ Carretera Iquitos Nauta km 4.5, Iquitos, Perú. E-mail: wernerchotam@yahoo.com \\ 2 Programa de Investigación Para el Uso del Agua y sus Recursos (AQUAREC), Instituto de Investigaciones de la Amazonía \\ Peruana (IIAP), Carretera Iquitos Nauta km 4.5, Iquitos, Perú. \\ 3 Institute de Recherche pour le Développement (IRD)-GAMET, UR175-CAVIAR, F-34000 Montpellier, France. \\ 4 Laboratoire Mixte International - Evolution et Domestication de l'Ichtyofaune Amazonienne (LMI - EDIA).
}

\section{RESUMEN}

La arahuana Osteoglossum bicirrhosum es un importante pez ornamental cuyas larvas y alevinos se exportan al mercado asiático. Esta alta demanda genera grandes presiones de pesca sobre sus poblaciones naturales, siendo necesario generar información genética de sus poblaciones que permitan elaborar planes de manejo para la especie. Para ello, diez loci microsatélites fueron analizados para evaluar la variabilidad genética de 299 individuos de arahuana en cinco localidades de la Amazonía peruana. Los resultados del dendrograma de agrupamiento según la distancia de Nei (1978) muestran que las localidades forman tres grupos principales (Bootstrap =91): Huicungo-El Dorado (Bootstrap=73), El Estrecho-Huapapa (Bootstrap $=73$ ) y Rimachi. Estas agrupaciones fueron corroboradas por el estimador $F_{s i}$, observándose diferenciación genética no significante únicamente entre las localidades del El Dorado versus Huicungo y El Estrecho versus Huapapa $\left(F_{s t}=0,05\right.$ y 0,07 respectivamente). Estos resultados muestran también un aislamiento genético por la distancia en línea recta entre pares de localidades, demostrando una dispersion de los peces preferencialmente por la llanura de inundación y no por el cauce de los ríos.

KEYWORDS: microsatélites, genética de poblaciones, peces ornamentales.

\section{FIRST STUDY OF THE GENETIC VARIABILITY OF SILVER AROWANA Osteoglossum bicirrhosum (Cuvier, 1829) IN THE LORETO REGION (PERUVIAN AMAZONIA)}

\begin{abstract}
The silver arowana Osteoglossum bicirrhosum is an important ornamental fish which larvae and young fish are exported to the Asian market. This high demand creates great fishing pressures on the natural populations, while it is necessary to generate genetic information of their populations enabling develop management plans of the specie. For this, ten microsatellite loci were analyzed to assess the genetic variability of 299 individuals of $O$. bicirrhosum in five localities in the Peruvian Amazon. The results of the clustering dendrogram (multivariate analysis built from distances of Nei, 1978) show that localities form three main groups (Bootstrap =91): Huicungo-El Dorado (Bootstrap=73), the Estrecho-Huapapa (Bootstrap $=73$ ) and Rimachi. These groups were corroborated by the estimator $\mathrm{F}_{\mathrm{st}}$, showing no significant genetic differentiation between localities El Dorado-Huicungo and El Estrecho-Huapapa $\left(\mathrm{F}_{\mathrm{st}}=0.05\right.$ and 0.07 , respectively), These results also show a genetic isolation by the straight-line distance between pairs of localities, demonstrating a dispersion of fish preferentially by the floodplain and not by the channel of the rivers.
\end{abstract}

PALABRAS CLAVE: microsatellites, population genetics, ornamental fish. 


\section{INTRODUCCIÓN}

La riqueza íctica de la cuenca amazónica hace que sea considerada entre las mayores proveedoras de peces ornamentales del mundo (Moreau \& Coomes, 2007). El 70\% del volumen de exportación sudamericana es cubierto por solamente 10 especies (Ortiz \& Iannacone, 2008), una de las cuales es la arahuana Osteoglossum bicirrhosum (Cuvier, 1829). La demanda de alevinos de $O$. bicirrhosum se incrementó debido a que esta siendo comercializado como sustituto de Scleropages formosus (especie filogenéticamente hermana de la arahuana), en los mercados de peces ornamentales asiáticos (Moreau \& Coomes, 2006), esto causa una reducción drástica de sus poblaciones naturales (Olivares et al., 2013). Ambas especies comparten características de su historia de vida que las hacen vulnerables a la sobreexplotación como la madurez tardía, baja fecundidad y cuidado parental bucal paterno (Duponchelle et al., 2012). En la Amazonía peruana, el sacrificio de machos adultos, sumado a un limitado monitoreo y deficiente control de su comercialización, ponen en peligro las poblaciones naturales de esta especie (Moreau \& Coomes, 2006; Da Silva et al., 2009). El comercio ornamental sobre las dos especies $O$. bicirrhosum y $O$. ferreirai en Amazonia colombiana tuvo como consecuencia su inclusión en el libro rojo de ese país, la primera como especie vulnerable y la segunda como especie en peligro de extinción (Argumedo, 2005).

$O$. bicirrhosum ha sido poco estudiado en la Amazonía peruana, desconociéndose muchos aspectos de sus rasgos de vida y diversidad genética de sus poblaciones; aspectos esenciales para desarrollar programas de manejo para su conservación (Balloux \& Lugon-Moulin, 2002). El presente trabajo pretende contribuir al conocimiento de la especie analizando la variabilidad genética de la arahuana $O$. bicirrhosum en los principales tributarios de la Amazonía peruana.

\section{METODOLOGÍA}

Se analizaron 299 individuos de arahuana procedentes de cinco localidades (Tabla 1): 58 en el lago Rimachi (Río Pastaza), 53 en el lago El Dorado (Río Yanayacu-Pucate), 53 en el lago Huicungo (Río Tapiche), 67 en El Estrecho y 68 en Huapapa (Río Putumayo). La extracción de DNA fue realizada mediante el protocolo CTAB modificado de Doyle \& Doyle (1987). Los 10 loci microsatélites analizados fueron tomados de Da Silva et al. (2009). Las amplificaciones fueron realizadas en volúmenes totales de $10 \mu$, conteniendo: $1 \mathrm{X}$ Buffer; $1,5 \mathrm{mM}$ $\mathrm{MgCl}_{2} ; 200 \mu \mathrm{M}$ de cada dNTP; $0,3 \mu \mathrm{M}$ de cada primer; $0,4 \mathrm{U}$ de Taq polimerasa y de 20 a $70 \mathrm{ng}$ de DNA genómico. Las condiciones de temperatura fueron: $94{ }^{\circ} \mathrm{C}$ x $1 \mathrm{~min}$ de denaturación inicial, seguido de 30 ciclos consistentes en: denaturación a $94^{\circ} \mathrm{C}$ x 20 s, hibridación a $60^{\circ} \mathrm{C}$ x $20 \mathrm{~s}$, y extensión a $72{ }^{\circ} \mathrm{C}$ x $20 \mathrm{~s}$; seguidos de una extensión final de $72{ }^{\circ} \mathrm{C}$ x $30 \mathrm{~min}$. La lectura de los microsatellites, fue realizada en un Analizador Genético Applied Biosystems 3130xl siguiendo la metodología del fabricante y los pesos de los alelos fueron determinados con el programa Peak Scanner versión 1.0. Con ayuda del software Genetix versión 4.05.2 fueron estimados entre pares de localidades el estimador del índice de fijación $F_{S T}$ (Weir \& Cockerham, 1984) usado para calcular la distancia genética de Rousset (1997), utilizada para determinar la correlación con las distancias geográficas entre pares de localidades siguiendo el cauce de los ríos o una línea recta entre localidades. Las distancia genética de Nei (1978) y la construcción del dendograma (Agrupamiento Vecino Próximo) con la prueba del bootstrap, fueron realizados con el software PHYLIP 3.5 (Felsenstein, 1993). La correlación entre la distancia genética y las distancia geográfica lineal y fluvial fueron estimadas a través del coeficiente de regresión de Pearson.

Tabla 1. Cuenca, localidad, georeferencias y número de ejemplares capturados (n) de arahuana Osteoglossum bicirrhosum colectados en la región Loreto, Amazonía peruana.

\begin{tabular}{llcc}
\hline \multicolumn{1}{c}{ Cuenca } & Localidad & Georeferencia & n \\
\hline Pastaza & Rimachi & $04^{\circ} 26.16$ S $76^{\circ} 40.01 \mathrm{~W}$ & 58 \\
Yanayacu-Pucate & El Dorado & $05^{\circ} 04^{\prime} 30^{\prime \prime} S 74^{\circ} 19^{\prime} 10^{\prime} \mathrm{W}$ & 53 \\
Tapiche & Huicungo & $06^{\circ} 07^{\prime} 53.0^{\prime \prime} \mathrm{S} 4^{\circ} 08^{\prime} 03.1^{\prime \prime} \mathrm{W}$ & 53 \\
Putumayo & El Estrecho & $02^{\circ} 35^{\prime} 1^{\prime \prime} \mathrm{S} 72^{\circ} 45^{\prime} 1^{\prime} \mathrm{W}$ & 67 \\
Putumayo & Huapapa & $02^{\circ} 32^{\prime} 20^{\prime} \mathrm{S} 70^{\circ} 25^{\prime} 34^{\prime} \mathrm{W}$ & 68 \\
\hline Total & & & $\mathbf{2 9 9}$ \\
\hline
\end{tabular}


Tabla 2. Índices de fijación $\left(F_{S T}\right)$ en el diagonal superior y distancia genética de Rousset (1997) en el diagonal inferior, estimados a partir de diez loci microsatélites para cinco poblaciones de arahuana Osteoglossum bicirrhosum en la región Loreto.

\begin{tabular}{lccccc}
\hline Poblaciones & Huicungo & El Dorado & Rimachi & El Estrecho & Huapapa \\
\hline Huicungo & - & $0,05^{* * *}$ & $0,22^{* * *}$ & $0,18^{* * *}$ & $0,22^{* * *}$ \\
El Dorado & 0,026 & - & $0,15^{* * *}$ & $0,07^{* * *}$ & $0,14^{* * *}$ \\
Rimachi & 0,100 & 0,066 & - & $0,17^{* * *}$ & $0,28^{* * *}$ \\
El Estrecho & 0,107 & 0,039 & 0,069 & - & $0,07^{* * *}$ \\
Huapapa & 0,120 & 0,073 & 0,124 & 0,032 & - \\
\hline
\end{tabular}

Significancia: ${ }^{* * *} p=0$

\section{RESULTADOS Y DISCUSIÓN}

Un total de 46 alelos fueron observados con los 10 loci microsatélites en los 299 individuos de $O$. bicirrhosum analizados. Los resultados del índice de fijación $\left(F_{S T}\right)$, la distancia genética de Rousset (1997 (Tabla 2), así como el dendrograma obtenido (con valores de Bootstrap elevados, obtenidos con 1000 réplicas) muestran una fuerte estructuración en la arahuana en la Amazonia peruana.

Esta estructuración está relacionada a las cuencas hidrológicas (Figura 1), y muestra que las arahuanas provenientes de las cinco localidades analizadas, están constituyendo tres poblaciones, originadas por una fuerte diferenciación genética significante entre estas agrupaciones.

Las poblaciones están conformadas por: Huicungo y El Dorado $\left(F_{S T}=0,05\right.$; Nei $=0,026$; Bootstrap $=73$ ), seguido de la población del Estrecho y Huapapa $\left(F_{S T}=0,07 ; \mathrm{Nei}=0,032\right.$; Bootstrap $=73$, mientras que la poblacion del lago Rimachi esta separada de todas las otras (Bootstrap = 91). Nuestros resultados muestran además, que la diferenciación genética está estructurada en relación con la distancia geográfica con una mayor correlación entre las distancias genéticas y las distancias geográficas lineales $(r=0,83)$ y una menor correlación entre las distancias genéticas y las distancias geográficas fluviales al largo de los ríos $(r=0,50)$ entre las cinco localidades de $O$. bicirrhosum estudiadas (Figuras 2A y 2B).

Nuestras observaciones de una estructura genética relacionada a la geografía son reforzadas por los obtenidos por Duponchelle et al. (2012), en el análisis de crecimiento de $O$. bicirrhosum en cuatro cuencas (Amazonas, Ucayali, Napo y Putumayo, esta última es la misma población de Huapapa que la de nuestro estudio), a través del estudio de otolitos, encontraron que hay una importante variabilidad en el crecimiento de la especie en las cuencas estudiadas. Según los autores esto puede ser resultado de la plasticidad fenotípica de la especie, debido a la heterogeneidad del medio ambiente o a la existencia de varias poblaciones distintas en Perú, que necesitan ser manejados independientemente. Otros estudios realizados por Waty (2009) y Ruiz (2011) también mostraron que rasgos de vida como la fecundidad, periodo de reproducción y crecimiento son variables entre poblaciones geográficas de $O$. bicirrhosum. Diferencias genéticas entre poblaciones, fueron ya reportados para $O$. bicirrhosum en la Amazonía colombiana por Bonilla et al. (2012), quienes mediante marcadores mitocondriales (COI y Cyt b) encontraron diferencias genéticas entre las poblaciones de arahuana provenientes de Tarapacá, Puerto Leguízamo, Leticia y La Pedrera. En Perú, la observación de una fuerte diferenciación genética entre poblaciones y un aislamiento genético por la distancia geográfica lineal y no por el cauce de los ríos, podrían estar relacionados: 1) al carácter relativamente sedentario de la arahuana (Pereira, 2007), en el sentido que la arahuana no realiza desplazamientos masivos cíclicos a través de los ríos con fines de reproducción o alimentación y 2) a una dispersión de la arahuana en busca de comida a través de la llanura de inundación en la época de creciente utilizando su capacidad de saltar fuera del agua (Saint-Paul et al., 2000).

En las arahuana asiaticas, Pouyaud et al. (2003) confirmaron la estructuración y diferenciación genética entre variedades de color en Scleropages formosus, a partir de secuencias nucleotídicas (Citocromo b), datos morfológicos, ecológicos y geográficos. Esto permitió describir tres especies nuevas especies ( $S$. macrocephalus, $S$. aureus y $S$. legendrei) dentro de la especie Scleropages formosus. 
Estos resultados también permitieron demostrar que existe una concordancia entre los datos genéticos y los parámetros ecológicos ( $\mathrm{pH}$ y color de agua) entre algunas especies de Arahuana asiática. Además, las relaciones entre los datos genéticos de las arahuanas asiáticas y los parámetros ecológicos fueron corroborados por Tang et al. (2004). La fuerte estructuración genética observada en $O$. bicirrhosum en la Amazonia peruana, seria principalmente originada por la distancia geográfica. Sin embargo como para sus especies hermanas de Asia, las barreras medioambientales (como las características físicoquímicas del agua) y la variación de las capacidades adaptativas entre las poblaciones (como la aptitud a la dispersión a través de la llanura), podrían reforzar la diferenciación genética en las arahuanas neotropicales. En efecto, Saint-Paul et al. (2000) mencionaron que las dos arahuanas sudamericanas, $O$. bicirrhosum y $O$. ferreirai (este último restricto a la cuenca del río Negro en Brasil) tienen una distribución alopátrica, asociada al tipo de agua: $O$. bicirrhosum habita en aguas neutras a ligeramente alcalinas, mientras $O$. ferreirai en aguas negras altamente ácidas. Además, reportes de desembarque pesquero y consulta con pescadores contribuyen a esta observación mostrando que $O$. bicirrhosum está ausente en las aguas ácidas del río Nanay $(\mathrm{pH}: 4,7$. Informe Técnico IRD-SENAMHI, 2002; Echenique et al., 2004). La hipótesis que los contrastes ecológicos entre los ríos, como por ejemplo entre el lago Rimachi (río Pastaza) y las localidades de Huapapa y El Estrecho (ambas en el río Putumayo), participan a la diferenciación genética intraespecifica de la Arahuana amazónica, debería ser probada en futuros estudios complementares.

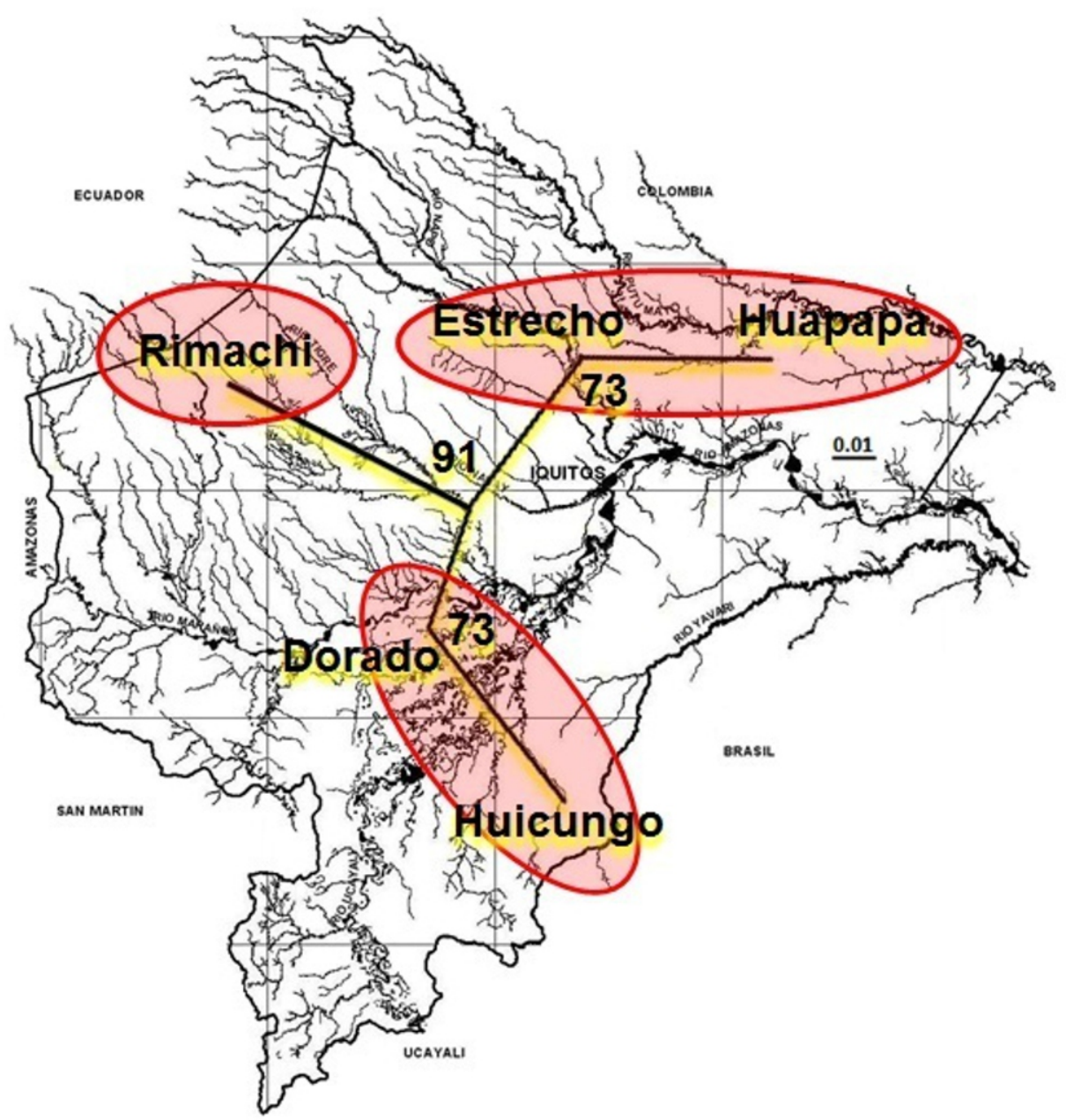

Figura 1. Dendrograma (insertado dentro del mapa de Loreto) construido a partir de las Distancias de Nei (1978) según el criterio de agrupamiento de vecino próximo para cinco localidades geográficas naturales de la arahuana Osteoglossum bicirrhosum en la región Loreto. 
(A)

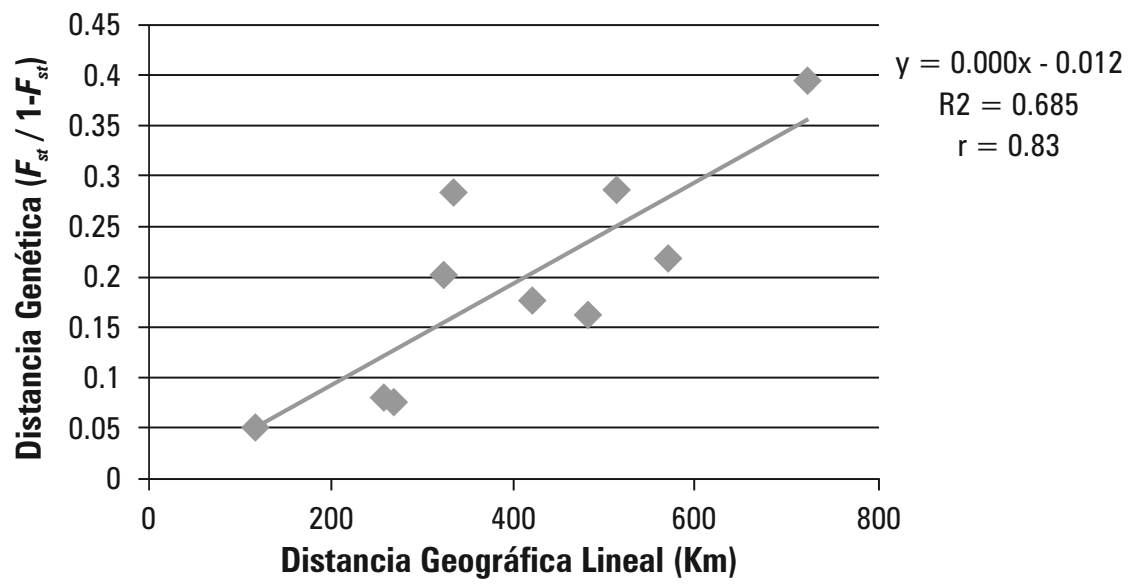

(B)



Figura 2. Representación gráfica del análisis de correlación entre la Distancia Genética de Rousset, $1997\left(F_{S T} / 1-F_{S T}\right)$ y Distancia Geográfica $(\mathrm{Km})$ Fluvial $(\mathrm{A})$ y Lineal $(\mathrm{B})$ obtenidos para cinco sitios de colecta de la arahuana Osteoglossum bicirrhosum en la región Loreto.

\section{BIBLIOGRAFÍA CITADA}

Argumedo, E. G. 2005. Arawanas, manual para la cría comercial en cautiverio. Asociación de Acuicultores del Caquetá (ACUICA). Caquetá,Colombia. 105pp.

Balloux, F.; Lugon-Moulin, N. 2002. The estimation of population differentiation with microsatellite markers. Molecular Ecology, 11: 155-165.

Da Silva, T. de J.; Hrbek, T.; Farias, I. P. 2009. Microsatellite markers for the silver arowana (Osteoglossum bicirrhosum, Osteoglossidae, Osteoglossiformes). Molecular Ecology Resources, 9 (3): 1019-1022.

Doyle, J. J.; Doyle J. L. 1987. A rapid AND isolation procedure for small quantities of fresh leaf tissue. Phytochem Bull, 19:11-15.

Duponchelle, F.; Ruiz, A.; Waty, A.; Panfili, J.; Renno, J-F.; Farfan, Farfan, F.; García-Vásquez, A.; CHU, F.; García-Dávila, C.; Vargas, G.; Ortiz, A.; Pinedo, R.; Núñez, J. 2012. Contrasted hydrological systems of the Peruvian Amazon induce differences in growth patterns of the silver arowana, Osteoglossum bicirrhosum. Aquat. Living Resour., 25: 55-66.

Echenique, R.O.; Núñez-Avellaneda, M.; Duque, S.R. 2004. Chlorococcales de la Amazonia colombiana I: Chlorellaceae y Scenedesmaceae. Caldasia, 26(1): 37-51. 
Felsenstein J. 1993. PHYLIP (Phylogeny inference pakage) versión 3.05 general information manual. University of Washintgton, Seattle, Washington. 132pp.

IRD-SENAMHI. 2002. Informe Técnico: "2da. Comisión de aforos, muestreo de agua y sedimentos - Cuencas de los Ríos Amazonas, Nanay, Marañón, Napo, Ucayali”.

Moreau, M. A.; Coomes, O. T. 2006. Potential threat of the international aquarium trade to silver arawana (Osteoglossum bicirrhosum) in the Peruvian Amazon. Oryx, 40: 1-9.

Moreau, M. A.; Coomes, O. T. 2007. Aquarium fish exploitation in western Amazonia: conservation issues in Peru. Environmental Conservation, 34(1): 12-22.

Ortiz, N.; Iannacone, J. 2008. Estado actual de los peces ornamentales amazónicos del perú que presentan mayor demanda de exportación. Biologist (Lima). Vol. 6, $\mathrm{N}^{\mathrm{o}}$ 1: 54-67.

Pereira, H. 2007. Estratégias reprodutivas de peixes aplicadas a aquicultura: bases para o desenvolvimento de tecnologías de producao. Rev Bras Reprod Anim, Vol. 31, N 3: 351-360.

Pouyaud. L.; Sudarto; Teugels, G. G. 2003. The different colour varieties of the Asian arowana Scleropages formosus (Osteoglossidae) are distinct species: morphologic and genetic evidences. Cybium, 27(4): 287-305.

Ruiz, A. C. 2011. Historia de vida de la "arahuana"
Osteoglossum bicirrhosum (cuvier, 1829) en tres cuencas de la amazonía peruana. Tesis para optar el grado académico de Magister en Zoología con mención Ecología y Conservación. Universidad Nacional Mayor de San Marcos. Lima-Perú. 63 pp.

Saint-Paul, U.; Zuanon, J.; Villacorta, M.; M. GARCIA, F. NOEMI. MARCH 2000. Fish communities in central Amazonian white- and blackwater floodplains. Environmental Biology of Fishes, 57(3): 235-250.

Tang, P. Y.; Sivananthan, J.; Pillay, S. O.; Muniandy, S. 2004. Genetic Structure and Biogeography of Asian Arowana (Scleropages formosus) Determined by Microsatellite and Mitochondrial DNA analysis. Asian Fisheries Society, 17 (2004): 81-92.

Waty, A. 2009. Etude des traits d'histoire de vie de cinq populations d'Arahuana (Osteoglossum bicirrhosum) subissant des pressions de pèches contrastées en Amazonie péruvienne. Université Montpellier II. Maestria en Ecologie et Biodiversité, spécialité en Biodiversité, Ecologie, Evolution. Montpellier. Francia. 10 p.

Weir, B.S.; Cockerham, C.C. 1984. Estimating Fstatistics for the analysis of population structure. Evolution, 38:1358-1370.

Recibido: 28 de Abril del 2016

Aceptado para publicación: 31 de Mayo del 2016 